\title{
Kaleidoscope laser properties and new optical fractal contexts
}

\author{
J. M. Christian ${ }^{1}$, G. S. McDonald ${ }^{1}$, A. S. Heyes ${ }^{1}$, J. G. Huang ${ }^{2}$ \\ ${ }^{1}$ Materials \& Physics Research Centre, University of Salford, Greater Manchester M4 4WT, UK \\ ${ }^{2}$ School of Engineering and Mathematical Sciences, City University London, London EC1V 0HB, UK
}

\begin{abstract}
We present the first detailed account of kaleidoscope laser modes where the equivalent Fresnel number $N_{\text {eq }}$ and magnification $M$ may assume arbitrary values. Properties of these linear fractal eigenmodes are explored through extensive numerical computations. Considerations are extended to demonstration and analyses of new contexts for spontaneous nonlinear optical fractals.
\end{abstract}

\section{KALEIDOSCOPE Fractal LASERS}

Unstable cavity lasers exhibit a plethora of bizarre and potentially exploitable phenomena that have fascinated researchers over the last four decades. In particular, the innate capacity of such simple systems for generating complex light patterns continues to attract wide and sustained interest. Within earlier collaborations [1,2], we discovered that the linear eigenmodes of one-dimensional (1D) and twodimensional (2D) unstable resonators are fractals - patterns that exhibit proportional levels of detail spanning many length scales. The fractality of these self-reproducing mode profiles was shown to originate from the interplay between round-trip cavity magnification, $M$, and periodic diffraction at the defining aperture of the system (e.g., a small feedback mirror) [3].

Kaleidoscope lasers are intuitive generalizations of classic unstable resonators to fully-2D transverse geometries, where the defining aperture has the shape of a regular polygon [3]. The non-orthogonal edges of this element have a profound impact on the structure of the cavity eigenmodes, which exhibit a striking level of beauty and complexity (see Fig. 1). Most obviously, $N$-sided regular-polygon boundary conditions impose $\mathrm{N}$-fold rotation symmetry on the intensity pattern. Kaleidoscope lasers offer enormous prospects for inspiring novel photonic device designs and architectures. For example, fractal light may prove to be more efficient for probing, scanning, writing and ablation applications in various technological, medical, research and industrial contexts.

Here, we present the first detailed analysis of kaleidoscope fractal lasers through accommodation of arbitrary equivalent Fresnel number $N_{\text {eq }}$ and magnification $M$. All previous analyses have been restricted to regimes where either $N_{\text {eq }}=$ $O(1)$ (in which case conventional ABCD matrix modelling can be deployed [4]) or $N_{\mathrm{eq}}>O(1)$ (where asymptotic approximations can be used [5]). Our approach is based on a fully-2D generalization of Southwell's Virtual Source method
[6], and exploits exact (Fresnel) mathematical descriptions of the constituent diffraction patterns [7].

One key advantage of our technique is that a single calculation allows one to access entire families of modes; another is that any particular mode may be computed to any desired accuracy. We also quantify the convergence properties of kaleidoscope laser modes (eigenvalue spectra and mode patterns themselves in the limit $N \rightarrow \infty$, where the feedback mirror becomes circular). The analysis employs a technique
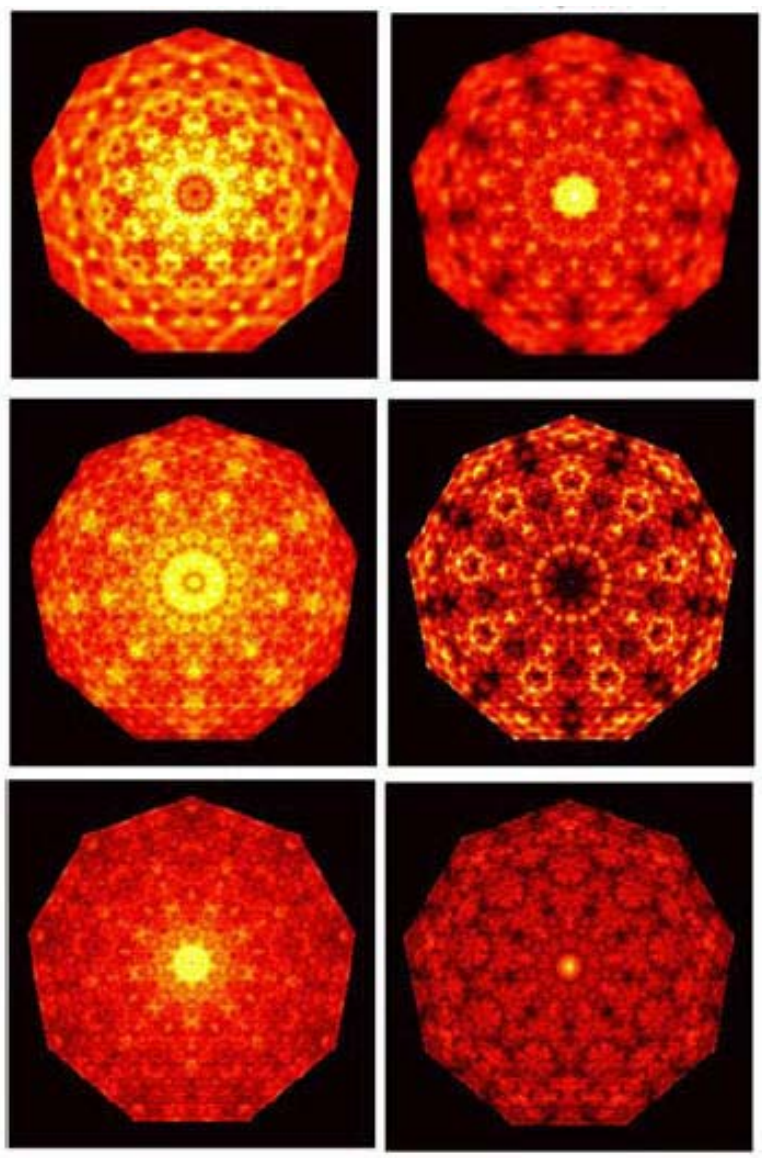

Fig. 1. Examples of lowest-order (column 1) and first-order (column 2) kaleidoscope fractal modes. Parameters are $N=9$ (nonagonal feedback mirror) and magnification $M=1.5$. Top row: $N_{\mathrm{eq}}=10$; middle row: $N_{\mathrm{eq}}=20$; bottom row: $N_{\mathrm{eq}}=30$. 
that unfolds each unstable cavity into an equivalent sequence of $N_{s}=\log \left(250 N_{\mathrm{eq}}\right) / \log (M)$ virtual apertures. Any eigenmode can then be constructed from a weighted superposition of the edge-waves diffracted by each aperture, plus a plane-wave contribution. In scaled units, the mode pattern $V(\mathbf{X})$ is given by

$$
V(\mathbf{X})=e_{0}\left[\frac{E_{N_{S}+1}\left(\mathbf{X}_{\mathbf{c}}\right)}{\alpha^{N_{S}}(\alpha-1)}+\sum_{m=1}^{N_{S}} \alpha^{-m} E_{m}(\mathbf{X})\right],
$$

where $\mathbf{X}$ denotes an appropriate set of transverse coordinates, $\mathbf{X}_{\mathbf{C}}$ is any point on the boundary of the feedback mirror, and $E_{m}(\mathbf{X})$ is the edge-wave pattern arising from the $m^{\text {th }}$ virtual aperture [7].

Since our interest lies with calculating the field over the feedback mirror, $e_{0}=+1$ if $\mathbf{X}$ lies inside this domain, and $e_{0}=0$ otherwise. The weighting factor $\alpha$, which plays the role of the mode eigenvalue, is obtained by finding the roots of an associated polynomial equation. A single virtual-source calculation provides information for determining the lowestloss mode and all the higher-order modes.

When $N \rightarrow \infty$, the feedback mirror becomes circular and the cavity essentially has only a single transverse dimension. This limit has been investigated by Berry under the assumption $N_{\text {eq }}$ $>>(1)$, and only for the lowest-loss mode [8]. For cavities with arbitrary $N_{\text {eq }}$ and $M$, this type of fully-2D convergence phenomenon does not lend itself to asymptotic analysis; indeed, it can only be truly addressed via numerical computation. We will present, to the best of our knowledge, the first in-depth treatment of the circular limit of families of kaleidoscope laser modes. It was found that this is a far more subtle problem than might first be imagined.

\section{New Optical Fractal CONTEXTS}

In later work [9], we examined whether nonlinear systems might give rise to spontaneous spatial fractals. It is well-

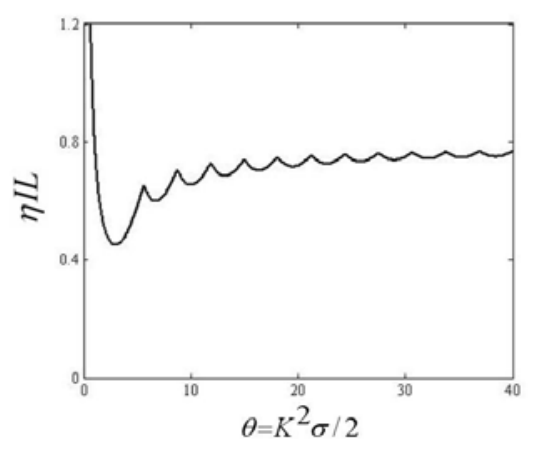

Fig. 2. A modulational instability threshold curve for two counterpropagating waves in a Kerr medium. The vertical axis is proportional to wave intensity, $I$, while the horizontal axis is involves the transverse wavenumber of the modulation, $K$. Other parameters are $\sigma$ (a transverse scale parameter), $L$ (the longitudinal interaction length), and $\eta= \pm 1$ (for a focusing or defocusing Kerr effect, respectively). known that nonlinear systems can exhibit a Turing instability in which homogeneous states develop large-amplitude emergent patterns from the smallest of fluctuations. However, such patterns are typically "simple", i.e. characterized by a single dominant length scale that corresponds to a single minimum in a modulational instability threshold plot. A range of nonlinear systems actually present a hierarchy of local Turing minima in their stability curves. It was proposed [9] that systems with such a multi-Turing property were capable of spontaneous spatial fractal formation. This hypothesis was tested, and confirmed true, for a single feedback mirror context involving a transient and diffusive nonlinear medium.

Here, we extend investigations to examine whether fractal pattern formation may arise from the one of the simplest possible configurations - the instantaneous interaction of two counter-propagating waves in a non-diffusive Kerr medium. This system [10,11] is known to present a multi-Turing modulational instability characteristic (see Fig. 2).

It will be shown, for the first time, that this fundamental configuration can also give rise to spontaneous fractal patterns. While we focus here on optical contexts, the implications of our findings extend to wave interactions in other (e.g. fluid and plasma) systems that are governed by the same pair of universal coupled nonlinear equations.
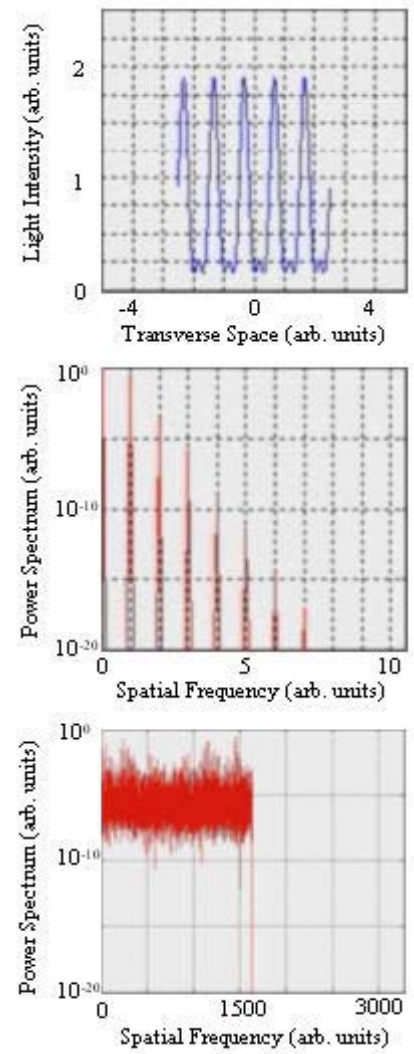

Fig. 3. Pattern formation in counter-propagating waves within a focusing Kerr medium. Top: spontaneous emergence of a simple transverse pattern. Middle: power spectrum of a simple pattern (involving a single dominant frequency and its harmonics). Bottom: power spectrum of a spontaneous spatial fractal (with a sharp cut-off at the carrier frequency). 
We will summarise investigations into the dependence of pattern formation character on various system parameters and confirm fractality of the resulting patterns through deployment of various appropriate fractal analysis measures.

Fig. 3 shows typical results for the counter-propagating waves system. By either: considering wave intensities close to the value dictated by the global instability minimum, or allowing for some spatial filtering mechanism in the system, simple patterns emerge. For higher wave intensities, and without strong spatial filtering, the system spontaneously generates fractal patterns with structure across decades of scale. The data shown in Fig. 3 corresponds to a nonlinear phase shift per medium transit of $\eta I L=0.8$. A simple pattern emerges when strong filtering is employed and fractal formation occurs when no spatial filtering is present. This result is consistent with the detail of the threshold curve plotted in Fig. 2.

More generally, it is found that depending on the precise parameters spontaneous spatial fractals can be either static (i.e.
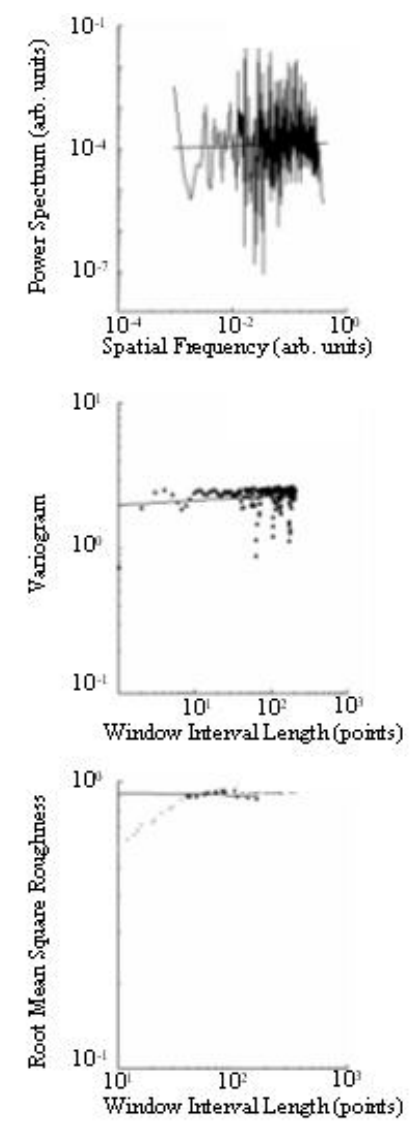

Fig. 4. Fractal analyses of spontaneous multi-scale pattern formation when $\eta I L=0.8$. Top: power spectrum fractal dimension determination, middle: the variogram method, bottom: roughness-length fractal dimension. Each measure confirms an area-filling curve of dimension close to 2 (for frequencies below the carrier wave cut-off frequency, and at length scales above the carrier wavelength). stationary) or dynamic in time. Also, for this particular system, each fractal generated has proved to be of a statistical and areafilling character (see Fig. 4).

Finally, we will briefly summarize our most recent results concerning spatial pattern formation in incoherent systems. The leading works in this field $[12,13,14]$ proposed that spatial pattern formation required either a perfectly coherent system or one involving a sufficiently non-instantaneous nonlinearity. However, subsequent work examining temporal pattern formation in fibre optic systems [15] reported that patterns may form irrespective of medium response time.

Two questions have been addressed concerning spatial pattern formation in incoherent systems involving instantaneous nonlinearity. Firstly, we have examined whether coherent spatial patterns emerge from initial states of high incoherence. Secondly, spatial pattern formation has been studied in systems that are maintained in states of partial incoherence. We will report on our finding that, within both of these contexts, spatial pattern formation is relatively robust.

\section{REFERENCES}

[1] G.P. Karman, and J.P. Woerdman, “ Fractal structure of eigenmodes of unstable-cavity lasers,” Opt. Lett., vol. 23, pp. 1909-1911, 1998.

[2] G.P. Karman, G.S. McDonald, G.H.C. New, and J.P. Woerdman, "Fractal modes in unstable resonators," Nature, vol. 402, pp. 138, 1999.

[3] G.H.C. New, M.A. Yates, G.S. McDonald, and J.P. Woerdman, "Diffractive origin of fractal resonator modes," Opt. Commun., vol. 193, pp. 261-266, 2001

[4] G.S. McDonald, G.P. Karman, G.H.C. New, and J.P. Woerdman, "Kaleidoscope laser," JOSA B, vol. 17, pp. 524-529, 2000.

[5] M. Berry, C. Storm, and W. van Saarloos, "Theory of unstable laser modes: edge waves and fractality," Opt. Commun., vol. 197, pp. 393-402, 2001.

[6] W.H. Southwell, "Virtual-source theory of unstable resonator modes," Opt. Lett., vol. 6, pp. 487-489, 1981.

[7] J.G. Huang, J.M. Christian, and G.S. McDonald, "Fresnel diffraction and fractal patterns from polygonal apertures," JOSA A, vol. 23, pp. 2768-2774, 2006.

[8] M. Berry, "Fractal modes of unstable lasers with polygonal and circular mirrors," Opt. Commun., vol. 200, pp. 321-330, 2001.

[9] J.G. Huang, and G.S. McDonald, "Spontaneous optical fractal pattern formation," Phys. Rev. Lett., vol. 94, art. no. 174101, 2005.

[10] W.J. Firth, and C. Pare, "Transverse modulational instabilities for counterpropagating beams in Kerr media," Opt. Lett., vol. 13, pp. 1096-1098, 1988.

[11] W.J. Firth, A. Fitzgerald, and C. Pare, "Transverse instabilities due to counterpropagation in Kerr media," J. Opt. Soc. Am. B, vol. 7, pp. 1087-1097, 1990

[12] D. Kip, M. Soljacic, M. Segev, E. Eugenieva, and N. Christodoulides, "Modulational instability and pattern formation in spatially incoherent light beams," Science, vol. 290, pp. 495-498, 2000.

[13] T. Schwartz, J.W. Fleischer, O. Cohen, H. Buljan, M. Segev, and T. Carmon, "Pattern formation in a ring cavity with temporally incoherent feedback," J. Opt. Soc. Am. B, vol. 21, pp. 2197-2205, 2004.

[14] T. Schwartz, T. Carmon, H. Huljan, and M. Segev, "Spontaneous patter formation with incoherent white light," Phys. Rev. Lett., vol. 93, art. no. 223901, 2004.

[15] A. Sauter, S. Pitois, G. Millot, and A. Picozzi, "Incoherent modulational instability in instantaneous nonlinear Kerr media," Opt. Lett., vol. 30, pp. 2143-2145, 2005. 